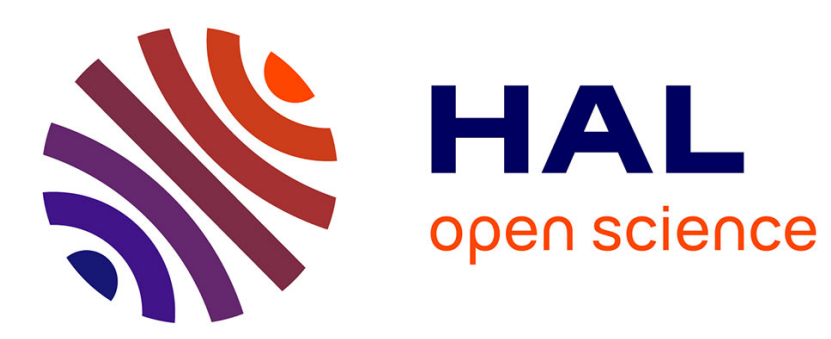

\title{
Multiscale DIC applied to Pantographic Structures
}

François Hild, A. Misra, F. Dell'Isola

\section{To cite this version:}

François Hild, A. Misra, F. Dell'Isola. Multiscale DIC applied to Pantographic Structures. Experimental Mechanics, 2021, 61, pp.431-443. 10.1007/s11340-020-00636-y . hal-02884633

\section{HAL Id: hal-02884633 \\ https://hal.science/hal-02884633}

Submitted on 30 Jun 2020

HAL is a multi-disciplinary open access archive for the deposit and dissemination of scientific research documents, whether they are published or not. The documents may come from teaching and research institutions in France or abroad, or from public or private research centers.
L'archive ouverte pluridisciplinaire HAL, est destinée au dépôt et à la diffusion de documents scientifiques de niveau recherche, publiés ou non, émanant des établissements d'enseignement et de recherche français ou étrangers, des laboratoires publics ou privés. 


\section{Multiscale DIC applied to Pantographic Structures}

\section{F. Hild · A. Misra · F. dell'Isola}

Received: date / Accepted: date

Abstract Background: Since the mechanical behavior of pantographic metamaterials depends upon the properties of their microstructure, accurate descriptions of unit cells are needed. Objective: The present effort is motivated by this requirement to characterize the detailed deformation of unit cells formed of two orthogonal sets of 3 beams. Methods: Their deformations in a bias extension test were measured via digital image correlation performed at different scales. Results: Thanks to the gray level residuals, the microscale results were found in better agreement with the experiment than mesoscale and macroscale analyses. Fine analyses around the hinges showed that relative displacements occurred between the

F. Hild*

Université Paris-Saclay, ENS Paris-Saclay, CNRS

LMT - Laboratoire de Mécanique et Technologie, 94235 Cachan, France

${ }^{*}$ Corresponding author. Email: francois.hild@ens-paris-saclay.fr

A. Misra

Civil, Environmental and Architectural Engineering Department, University of Kansas, 1530 W. 15th Street, Learned Hall, Lawrence, KS 66045-7609, USA

F. dell'Isola

MEMOCS and DICEEA Università dell'Aquila, DISG Università di Roma La Sapienza, Italy 
two beam layers. Conclusions: Such experimental analyses supply full-field data to validate models (e.g., as a starting point in homogenization procedures) for describing the mechanical behavior of pantographic metamaterials.

Keywords Digital Image Correlation (DIC) - Finite element · Gray level residuals ·

Metamaterial $\cdot$ Multiscale analyses

\section{Introduction}

At least three different characteristic scales can be distinguished when studying pantographic structures [4]. The description at the microscale considers the metamaterial as constituted by a standard Cauchy continuum whose shape is detailed as the union of rectangular and circular cylinders (see Figure 1 (b)). At this scale, the detailed deformation of the considered body is fully described. The material is geometrically complex but its constitutive equations are those of a simple first gradient isotropic continuum. At the mesoscale, the metamaterial may be considered as made of elastic, extensional and flexible beams interconnected by possibly elastic constraints. At the macrolevel, it is possible to introduce, as a predictive model, a second gradient continuum whose geometry is particularly simple. However, it is governed by more complex deformation energy functionals.

The pantographic structure that is considered herein is characterized, at the mesoscale, by two families of equally spaced beams interconnected at their intersection points by means of deformable cylinders. The question addressed herein concerns the description of the deformation phenomenology involving these cylinders, namely, can they be modeled as hinges (i.e., constraints prescribing equality of displacements and allowing for relative rotations) possibly with a deformation energy related to these rotations? Or is it necessary to assume that relative displacements occur at these interconnection points? 
Structures constituted by the pantographic cells shown in Figure 1 can be fabricated via additive manufacturing (3D printing) [5]. They supply an interesting example of metamaterials synthesizing second gradient continua [26,4]. Further, these material systems undergo large deformation ( $>50 \%$ stretch) while remaining elastic. In recent years, refined mathematical models have been proposed to replicate such behavior [23,8]. They can be classified as either continuum or discrete models. The necessary continuum models for describing the complex kinematics of these metamaterials are those in which the strain energy depends upon the second gradient of deformation. Conversely, discrete Hencky spring-link models offer an alternative approach for describing the deformation of pantographic systems. Qualitative comparisons of the predictions obtained with both discrete and continuous models with experimental results were carried out [23,26].

Since the mechanical behavior of pantographic structures at the macroscale depends upon the properties of their microstructure, accurate mathematical description of pantographic unit cells is needed. The present effort is motivated by this requirement to characterize the detailed deformation of unit cells formed of two orthogonal sets of 3 beams. They were subjected to a tensile experiment and digital image correlation (DIC) was conducted to extract the details of their deformation.

DIC consists in registering a series of images acquired during mechanical tests [20, 19]. From such analyses, displacement, strain and residual fields are obtained. When dealing with pantographic metamaterials, FE-based DIC was applied at the macroscale [26] and mesoscale [4] for which the mesh was tailored to the pantograph surface with simple morphological operations. In both cases, regularized DIC [16,22] was selected since the random contrast was very weak in the analyzed experiments. An alternative route consists in backtracking, via regularized DIC, nominal meshes to fit the experimental reference configuration [5]. Both approaches will be used herein. 
For the experiment reported herein, FE-based DIC was applied at three different scales. Hencky-type regularization (i.e., incremental elastic regularization) was implemented. Thanks to such type of regularization, large strains can be captured without penalizing too much the registration scheme. Further, focused analyses about the hinges will also be carried out via local and global DIC at the microscale.

\section{Multiscale DIC}

It has been previously noted that the considered mechanical system is characterized by its multiscale structure. It is therefore natural to perform DIC analyses at different scales. In the pantographic metamaterial considered herein, meshes using 3-noded finite elements were constructed. The interpolation functions are then linear within each element, and the nodal displacements become the unknowns to be determined by minimizing the sum of squared gray level residuals [12,17] over the considered region of interest (ROI)

$$
\varphi_{c}^{2}(\{\boldsymbol{v}\})=\sum_{\mathrm{ROI}} \rho^{2}(\boldsymbol{x})
$$

with respect to the nodal displacements $v_{i}$, which are gathered in the column vector $\{\boldsymbol{v}\}$, such that the displacement $\boldsymbol{u}$ of any material point $\boldsymbol{x}$ reads

$$
\boldsymbol{u}(\boldsymbol{x})=\sum_{i} v_{i} \boldsymbol{N}_{i}(\boldsymbol{x})
$$

where $\boldsymbol{N}_{i}$ are finite element shape functions. The pixel-wise gray level residual reads

$$
\rho(\boldsymbol{x})=f(\boldsymbol{x})-g(\boldsymbol{x}+\boldsymbol{u}(\boldsymbol{x}))
$$

where $f$ is the picture in the reference configuration, $g$ the picture in the deformed configuration. The root mean square (RMS) of $\rho$ computed over the ROI defines the overall registration quality associated with any selected kinematic basis of measured displacement fields. 
The minimization of $\varphi_{c}^{2}$ is performed via Gauss-Newton iterations

$$
\left[\boldsymbol{H}_{\boldsymbol{c}}\right]\{\partial \boldsymbol{v}\}=\left\{\boldsymbol{h}_{\boldsymbol{c}}\right\}
$$

where $\left[\boldsymbol{H}_{\boldsymbol{c}}\right]$ denotes the DIC Hessian, $\left\{\boldsymbol{h}_{\boldsymbol{c}}\right\}$ the DIC residual vector. These two quantities are obtained by assembly of elementary Hessians for each element $e$

$$
\left(H_{c}^{e}\right)_{j k}=\sum_{\Omega^{e}}\left(\boldsymbol{\nabla} f(\boldsymbol{x}) \cdot \boldsymbol{N}_{j}(\boldsymbol{x})\right)\left(\boldsymbol{\nabla} f(\boldsymbol{x}) \cdot \boldsymbol{N}_{k}(\boldsymbol{x})\right)
$$

and elementary DIC vectors

$$
\left(h_{c}^{e}\right)_{j}=\sum_{\Omega^{e}}(f(\boldsymbol{x})-g(\boldsymbol{x}+\widetilde{u}(\boldsymbol{x})))\left(\boldsymbol{\nabla} f(\boldsymbol{x}) \cdot \boldsymbol{N}_{j}(\boldsymbol{x})\right)
$$

where $\Omega^{e}$ is the part of the ROI covered by element $e$, and $\widetilde{u}$ corresponds to the current estimate of the displacement field. The minimization scheme consists in gradually correcting the successive estimates $\{\widetilde{\boldsymbol{v}}\}$ of the total nodal displacement vector $\{\boldsymbol{v}\}$. After each iteration, the image $g$ corrected by the measured displacement field $\widetilde{u}(i . e ., g(\boldsymbol{x}+\widetilde{u}(\boldsymbol{x})))$ is updated for any pixel $\boldsymbol{x}$ belonging to the ROI. The DIC vectors are subsequently re-evaluated and a new iteration starts. In the present setting, the DIC Hessian is computed once for all since it only depends on the gradient of the picture in the reference configuration (Equation (5)).

The DIC functional is penalized with a second one [21], which is constructed by computing the norm of the nodal forces for Hencky elasticity (i.e., minimizing the equilibrium gap [3] written in terms of the incremental displacements $\{\delta \boldsymbol{v}\}$ between two consecutive pictures [5])

$$
\varphi_{m}^{2}(\{\boldsymbol{v}\})=\{\boldsymbol{\delta} \boldsymbol{v}\}^{\top}[\boldsymbol{K}]^{\top}[\boldsymbol{K}]\{\boldsymbol{\delta} \boldsymbol{v}\}
$$

The minimization of $\varphi_{t}^{2}=\varphi_{c}^{2}+w_{m} \varphi_{m}^{2}$ is performed via Gauss-Newton iterations

$$
\left[\boldsymbol{H}_{c m}\right]\{\partial \boldsymbol{v}\}=\left\{\boldsymbol{h}_{\boldsymbol{c}}\right\}-w_{m}[\boldsymbol{K}]^{\top}[\boldsymbol{K}]\{\boldsymbol{\delta} \widetilde{\boldsymbol{v}}\}
$$


with

$$
\left[\boldsymbol{H}_{c m}\right]=\left[\boldsymbol{H}_{\boldsymbol{c}}\right]+w_{m}[\boldsymbol{K}]^{\top}[\boldsymbol{K}]
$$

where $\{\delta \widetilde{\boldsymbol{v}}\}$ denotes the current estimate of the incremental displacement, and $[\boldsymbol{K}]$ the stiffness matrix. The weight of this functional $w_{m}$ is proportional to a length $\ell_{\text {reg }}$ raised to the power 4 [16,22]. With such regularization scheme, there are two length scales, namely, one associated with the element size $\ell$, and another one with the mechanical regularization (i.e., $\ell_{\text {reg }}$ ). This length defines the physical size of the zone over which Hencky elasticity applies.

Last, kinematic constraints will be added to deal with perfect hinges (i.e., the displacement of the top and bottom beam will be assumed identical at the hinge axis, and the corresponding rotation is free). In the present case, Lagrange multipliers, which are gathered in the column vector $\{\boldsymbol{\lambda}\}$, characterize pseudo-cohesive forces (associated with the DIC formulation) that need to be applied to satisfy the considered constraint (i.e., no displacement jump at the hinges). The minimization procedure then consists in iteratively solving

$$
\left[\begin{array}{cc}
{\left[\boldsymbol{H}_{c m}\right]} & {[\boldsymbol{L}]^{\top}} \\
{[\boldsymbol{L}]} & {[\mathbf{0}]}
\end{array}\right]\left\{\begin{array}{c}
\{\boldsymbol{\partial} \boldsymbol{v}\} \\
\{\boldsymbol{\partial} \boldsymbol{\lambda}\}
\end{array}\right\}=\left\{\begin{array}{c}
\left\{\boldsymbol{h}_{\boldsymbol{c}}\right\}-w_{m}[\boldsymbol{K}]^{\top}[\boldsymbol{K}]\{\boldsymbol{\delta} \widetilde{\boldsymbol{v}}\} \\
\{\mathbf{0}\}
\end{array}\right\}
$$

where $[\boldsymbol{L}]$ gathers all constraint equations written on nodal displacements of different parts of the mesh.

Once any of the previous Hessians is available, the corresponding DIC vectors are iteratively computed as the nodal displacements are updated. The convergence criterion consists in having the L2-norm of the nodal displacement increments $\{\delta \boldsymbol{v}\}$ become less than $10^{-3} \mathrm{px}$, which, given the very large amplitude of the displacements of the reported experiment (i.e., greater than $220 \mathrm{px}$ ), is very severe. All results reported hereafter were obtained after such convergence was achieved. 
The DIC analyses were performed using the Correli 3.0 framework [11] in which the above described elastic regularization was implemented (Table 1 ). With the selected regularization length (i.e., $\ell_{\text {reg }}=150 \mathrm{px}$ ), the standard displacement uncertainty was less than $0.005 \mathrm{px}$ irrespective of the various meshes that will be analyzed herein (i.e., their characteristic sizes were always lower than the regularization length, so that the measurement uncertainty is controlled by $\ell_{\text {reg }}$ ). These results were obtained by registering the first two images acquired during the experiment for which the crosshead of the testing machine did not move.

Table 1 DIC analysis parameters

\begin{tabular}{|l|l|}
\hline \hline DIC software & Correli $3.0[11]$ \\
Image filtering & none \\
Element length (mean) & 12,10 and $11 \mathrm{px}$ \\
Shape functions & linear (T3) \\
Mesh & see Figures 2 d), 3 b) and 4 b-c) \\
Matching criterion & penalized sum of squared differences \\
Regularization length & 150 px \\
Interpolant & cubic \\
Displacement noise-floor & $<0.005 \mathrm{px}$ \\
\hline \hline
\end{tabular}

\section{Multiscale Meshes}

The analyses performed herein involved different scales for the description of the kinematics of pantographic cells. Such descriptions were tailored to the corresponding models that may be developed. Macroscale registrations utilized simple discretizations of the kinematics in which the local details of the microstructure were not accounted for (i.e., the solid phase and air are meshed). In mesoscale analyses, the external morphology of the solid phase was 
described. However, the internal kinematics was approximated by continuous displacement fields. Conversely, the local details of the internal kinematics associated with the hinges was investigated in microscale studies. For each of these scales, different meshes based on 3-noded elements were constructed.

\subsection{Millimetric Pantograph}

The specimen considered in this analysis was designed to represent the unit cell behavior of pantographic metamaterials. Their geometries were generated using the CAD software SolidWorks (Dassault Systems SolidWorks Corporation, Waltham, MA, USA). The samples were fabricated using the 3D printer Formiga P 100 (EOS GmbH, Munich, Germany). The printer used the SLS (selective laser sintering) technology to fabricate pantographic unit cells using polyamide powder (PA2200) with average grain size of $56 \mu \mathrm{m}$. Figure 1 gives a schematic view of the studied structure, the pantographic unit cell indicating the geometrical parameters, and the printed sample. For the tested specimen, the geometrical parameters were as follows: $p=13 \mathrm{~mm}, L=39 \mathrm{~mm}, a=b=1 \mathrm{~mm}, d=0.9 \mathrm{~mm}, h=2 \mathrm{~mm}, \theta=90^{\circ}$, and $\phi=45^{\circ}$.

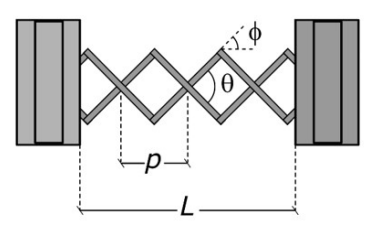

(a)

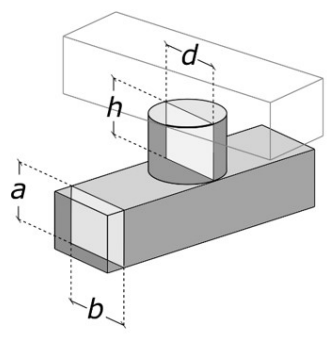

(b)

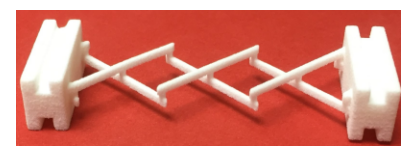

(c)

Fig. 1 (a) Schematic view of the pantographic structure studied herein, and (b) corresponding unit cell. Printed cells ( $p=13 \mathrm{~mm}, L=39 \mathrm{~mm}, a=b=1 \mathrm{~mm}, d=0.9 \mathrm{~mm}, h=2 \mathrm{~mm}, \theta=90^{\circ}, \phi=45^{\circ}$ ) 


\subsection{Macroscopic Meshes}

First, a series of macroscopic meshes encompassing the pantographic structure was considered (Figure 2). In the present case, structured meshes were created from nodal sequences of a rectangular ROI. Four different discretizations were constructed, namely for average element sizes $\ell$ ranging from 90 px for the coarsest mesh down to 12 px for the finest mesh. The element size was defined as the square root of the mean element surface for any considered mesh. The sought kinematics thus corresponded to the macroscopic response of such materials.

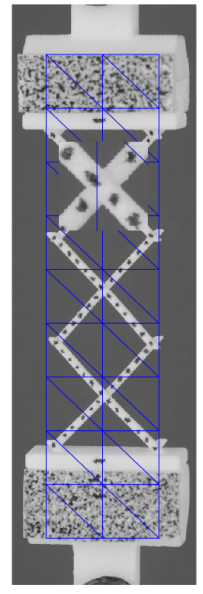

(a) $\ell=90 \mathrm{px}$

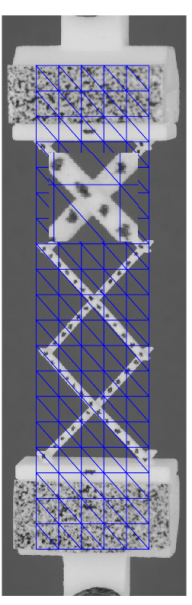

(b) $\ell=39 \mathrm{px}$

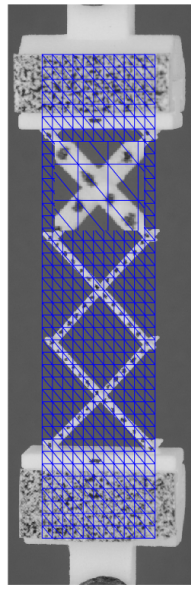

(c) $\ell=18 \mathrm{px}$

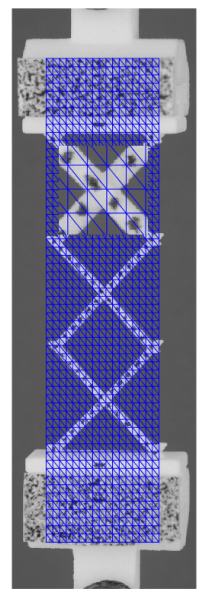

(d) $\ell=12 \mathrm{px}$

Fig. 2 Different meshes used in DIC analyses at the macroscale. The reference configuration of the pantographic metamaterial is also shown. The insets show meshing details of the underlying hinge

\subsection{Mesoscopic Meshes}

Second, FE-based DIC registrations were also performed at the mesoscale. In those cases, the external geometry of the pantographic structure was explicitly accounted for, but the kinematics was that of an underlying FE mesh in which the hinges were not explicitly de- 
scribed (Figure 3). The mesh was tailored to the pantograph surface via simple morphological operations [4] in order to create a mask of the solid phase. Each boundary was then interpolated using B-splines to avoid its pixellization. These contours were used to prepare the geometrical features (i.e., points, lines, line loops and surfaces) as input to GMSH [6] in which the mesh density can be varied at will. Three different discretizations were chosen (i.e., average element sizes $\ell$ ranging from $20 \mathrm{px}$ down to $5 \mathrm{px}$ ).

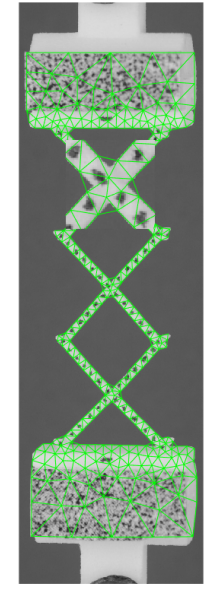

(a) $\ell=20 \mathrm{px}$

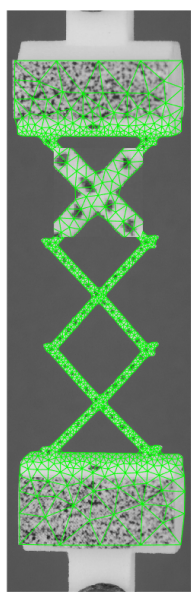

(b) $\ell=10 \mathrm{px}$

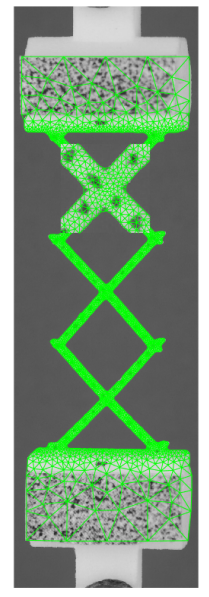

(c) $\ell=5 \mathrm{px}$

Fig. 3 Different meshes used in DIC analyses at the mesoscale. The insets show meshing details of the underlying hinge

\subsection{Microscopic Meshes}

To account for the 11 hinges (Figure 4(a)), a third (i.e., microscopic) mesh was constructed. Each beam of the pantograph was individually meshed (Figure 4 b)) with a mean element size $\ell=11$ px. Consequently, the two meshes overlapped in the intersection areas about each hinge. Another option for specifically tracking the hinge motions was to mesh small zones of interest centered about each hinge (Figure 4(c)). In the present case, four 11-px 
triangles were centered about each hinge to form a square window. From such analyses, the first order kinematics of the pantograph (i.e., the motions of the hinges) was measured. This type of analysis is typically suited for discrete models [23 25]. For such local approaches, the elastic regularization was not as effective, which led to a standard uncertainty less than $0.025 \mathrm{px}$. This level was greater than for the previous cases (Table 11, yet still very low for a DIC analysis with such small interrogation windows.

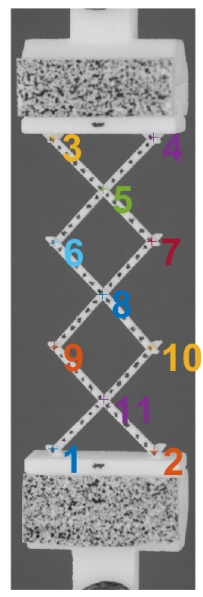

(a)

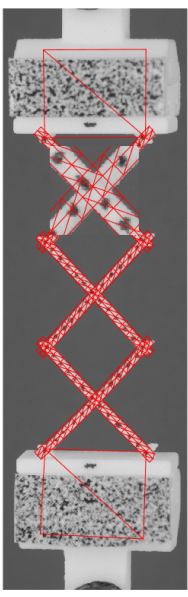

(b)

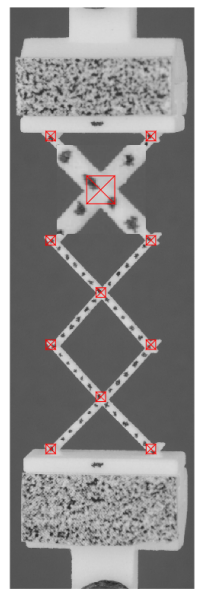

(c)

Fig. 4 Meshes used in DIC analyses at the microscale $(\ell=11 \mathrm{px})$. (a) Location and labels of the 11 hinges. (b) Meshed beams for global analysis. (c) Zones of interest centered about each hinge for the local approach. The insets show meshing details of the underlying hinge

To obtain the mesh shown in Figure 4(b), an additional operation was needed, namely backtracking the mesh constructed on the nominal geometry of the pantograph. Figure 5 (a) shows the initial configuration composed of two series of beams (in blue), and the two supports (in red and green). The supports were only coarsely meshed as the only relevant information is the displacement of the four connections with the beams (i.e., hinges \#1, 2, 3 , 4). From this mesh, a mask was created for which the gray level was equal to the average 
gray level of the pantograph. The background had a uniform gray level identical to that of the true experiment.

This first mesh was backtracked to fit as best as possible the actual geometry via regularized DIC. An auxiliary and structured (yellow) mesh was constructed on the picture in the reference configuration (Figure 5(b)). With this mesh, the experimental configuration (Figure 5 b)) was registered with respect to the nominal configuration (Figure 5 a)). At convergence, the nominal mesh (Figure 5(c)) was then backtracked by moving back all nodes (Figure 5 d)). A very good match is observed.

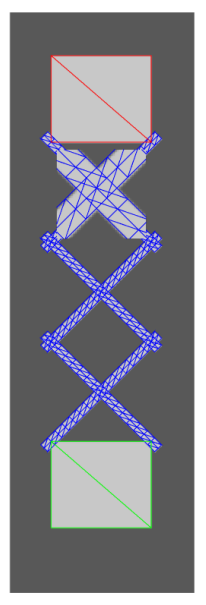

(a)

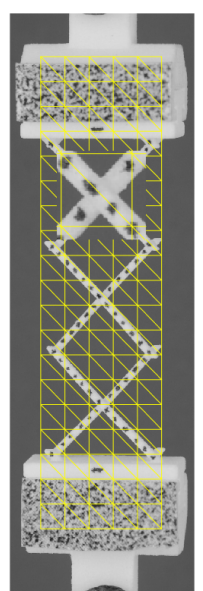

(b)

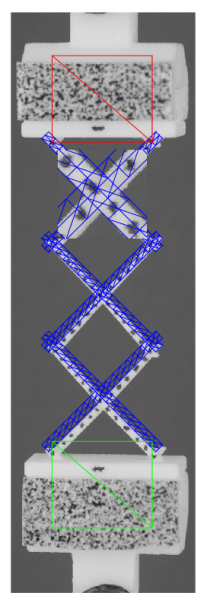

(c)

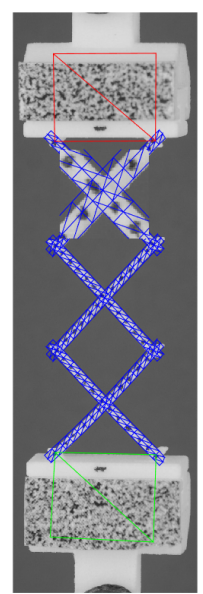

(d)

Fig. 5 Procedure of mesh backtracking. (a) Nominal mesh over the mask. (b) Experimental reference configuration and auxiliary mesh. (c) Nominal mesh laid over the picture of the reference configuration. (d) Backtracked mesh laid over the picture of the reference configuration. The insets show meshing details of the underlying hinge

The perfect hinges were then recreated by using Lagrange multipliers prescribing the corresponding kinematic constrains (in terms of nodal displacements) in the registration systems to solve (see Equation (10). The beams were therefore allowed to rotate about each 
hinge, which was not possible in the mesoscopic analyses. The two supports of the pantographs were also meshed with two triangular elements and connected to the corresponding hinges of the pantograph.

The choice of any discretization was probed by computing the correlation residual field $\rho$ (Equation (3) and its RMS level over the ROIs of any DIC calculation. The lower the residual, the more trustworthy the measured displacement field. Given the fact that the three scales did not consider the same region of interest, the gray level residuals of the macroscopic analyses cannot be directly compared to those at the meso- and micro-scales.

\section{Analysis of Tensile Test}

The pantographic unit cell specimen was subjected to tensile loading in step-by-step quasistatic conditions. The experimental setup and testing procedure are briefly described here for completeness (see for other details in Refs. [14,27]). The experiment was performed using Bose ElectroForce 3200 (now TA Instrument) testing device controlled by the software WinTest Material Testing System. Reaction forces were measured using a load cell with measurement range of $\pm 22 \mathrm{~N}$, a standard measurement uncertainty of $0.1 \%$, and a noise floor of $0.001 \mathrm{~N}$. The test was conducted in displacement control mode with a crosshead velocity of $0.1 \mathrm{~mm} / \mathrm{s}$, where the displacement was measured using the built-in transducer with the range of $\pm 6.5 \mathrm{~mm}$, a standard measurement uncertainty of $0.1 \%$ and a noise floor of $1 \mu \mathrm{m}$. The grips connecting the specimen to the loading machine were designed to apply an axial tensile action and resist the overall specimen rotation about the loading axis. Consequently, the specimen was restricted from Poynting effect related to specimen rotation about the loading axis observed for pantographic metamaterials under stretch [13]. The crosshead displacement amplitude was $12 \mathrm{~mm}$. 
For evaluations of the displacement fields, the specimen grips were sprayed on their surface to make a black speckle pattern prior to the testing. However the surfaces of the beams were just marked by a set of crosses whose distance was about 30 px. Such situation required the regularization to be adapted and a size of $\ell_{\text {reg }}=150$ px enabled all DIC calculations reported herein to converge. The hardware parameters of the optical setup are reported in Table 2 A series of 80 pictures was acquired when one loading and unloading cycle was performed.

Table 2 DIC hardware parameter

\begin{tabular}{|l|l|}
\hline \hline Camera & NIKON D300 \\
Definition & $4288 \times 2848$ pixels (RGB image) \\
Gray Levels amplitude & 8 bits \\
Lens & AF-S VR Micro-Nikkor 105mm f/2.8G ED \\
Aperture & $f / 4.5$ \\
Field of view & $74 \times 111 \mathrm{~mm}^{2}$ \\
Image scale & $52 \mu \mathrm{m} / \mathrm{px}$ (B\&W image) \\
Stand-off distance & $\approx 40 \mathrm{~cm}$ \\
Image acquisition rate & $1 / 3 \mathrm{fps}$ \\
Exposure time & $20 \mathrm{~ms}$ \\
Patterning technique & Sprayed black paint and black crosses \\
Pattern feature distance & $30 \mathrm{px}$ (crosses) \\
\hline \hline
\end{tabular}

Figure 6 shows three particular images of the test, namely, the reference configuration, one picture at the middle of the loading cycle, and the last of the loading cycle. This case is deemed challenging for DIC for the following reasons. First, there was virtually no pattern on the beams. As mentioned above, this observation explains why regularization had to be implemented. Otherwise, only the black features could have been tracked. Second, the 
pantographic metamaterial deformed a lot. Third, because of the pantographic nature of the metamaterial, material points gradually disappeared since the motion of two superimposed layers of beams were analyzed. Last, but not least, the local kinematics of such metamaterials was complex.

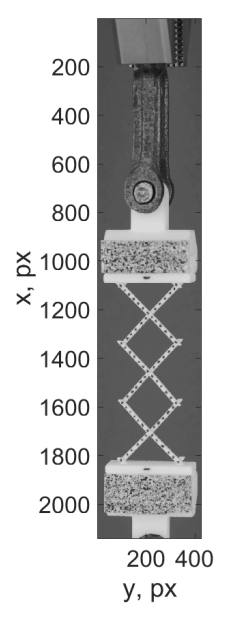

(a)

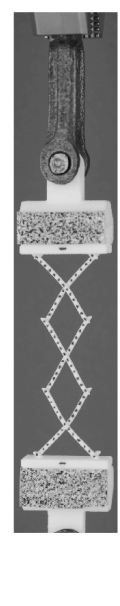

(b)

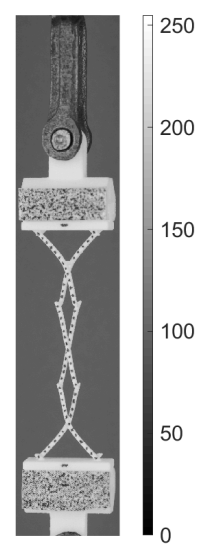

(c)

Fig. 6 Gray level images of the reference configuration (a, image \#0), and two deformed configurations at the middle (b, image \#19) and at the end (c, image \#39) of the loading ramp

Figure 6 also shows the reference frame associated with matrices of pixels. All results reported hereafter will refer to this frame.

\subsection{Macroscale Results}

Figure 7 shows the change of the correlation residual (i.e., $\operatorname{RMS}(\rho)$ ) for the four different meshes used in macroscopic registrations (Figure 2). The higher the applied displacement, the higher the residuals for all meshes. Further, there is a clear difference between the correlation residual for the coarse mesh (i.e., average of $\operatorname{RMS}(\rho)$ was equal to 21 gray levels) and that of the finest discretization (i.e., average of $\operatorname{RMS}(\rho)$ reduced to 14 gray levels). 
The 12-pixel discretization led to residuals very close to the 18-px mesh (i.e., average of $\operatorname{RMS}(\rho)$ equal to 15 gray levels). Both meshes (i.e., $\ell=12,18 \mathrm{px}$ ) are therefore considered to be equivalent in this first series of tests.

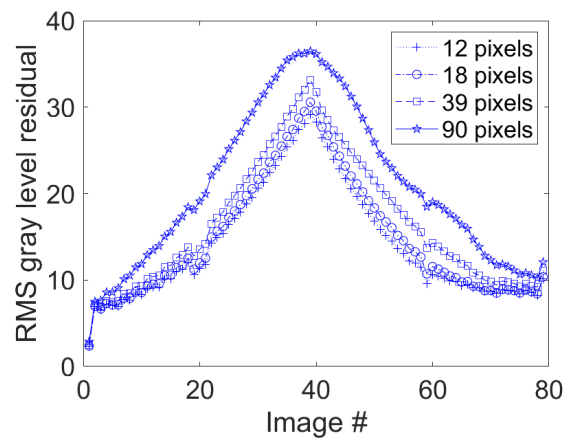

Fig. 7 RMS gray level residuals in DIC analyses at the macroscopic scale

The RMS residuals observed in this first case were very high in comparison with generally accepted levels in DIC analyses [9], which is typically of the order of one percent of the dynamic range (i.e., ca. 2-3 gray levels for 8-bit pictures and conventional cameras). This is only the case for the first picture pair for which the test had not started. Early on, the fact that the residuals are higher is an indication that the kinematic hypotheses are not fully consistent with the experimental reality, and proves that DIC analyses were very challenging in the present case. Last, let us note that the RMS variations for pictures 19-21 and 59 were due to lighting variations. Brightness and contrast corrections could have been applied [9]2]; they were not because these fluctuations remained sufficiently low in comparison with other sources of error. The small increase for the last picture (79) was due to small contractions of the metamaterial (starting at picture 72). 
To better understand these differences, Figure 8 a-d) shows the gray level residual fields for the 19-th picture (i.e., corresponding to about half the maximum prescribed displacement).

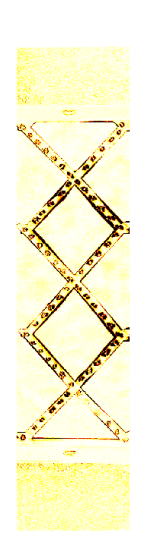

(a) $\ell=90 \mathrm{px}$

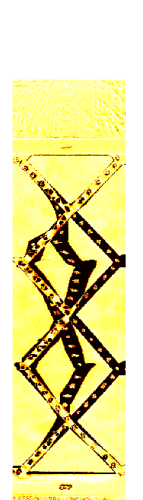

(e) $\ell=90 \mathrm{px}$

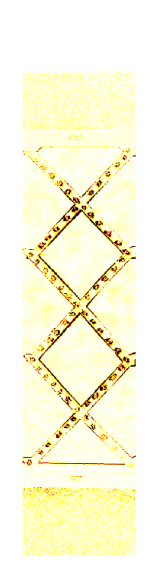

(b) $\ell=39 \mathrm{px}$

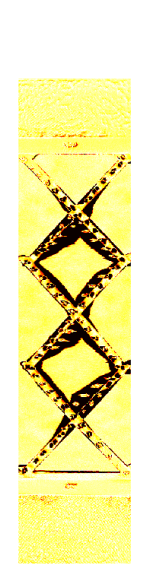

(f) $\ell=39 \mathrm{px}$

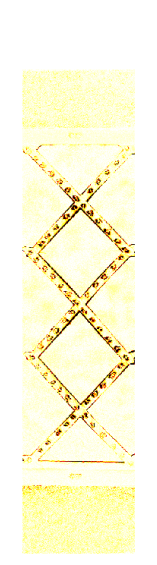

(c) $\ell=18 \mathrm{px}$

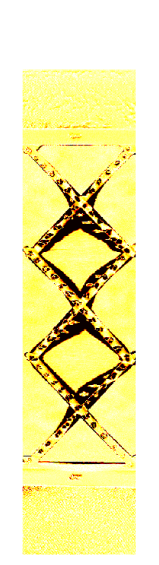

(g) $\ell=18 \mathrm{px}$

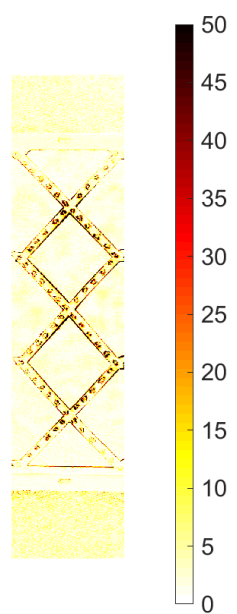

(d) $\ell=12 \mathrm{px}$

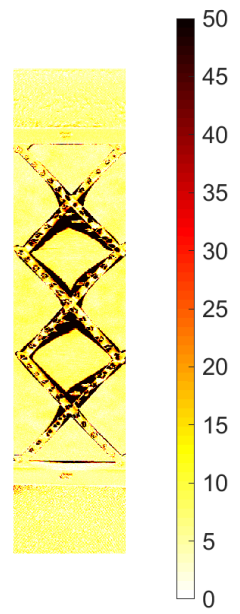

(h) $\ell=12 \mathrm{px}$

Fig. 8 Gray level residual fields for the four different meshes used in DIC analyses at the macroscale of the 19-th (top row) and 39-th (bottom row) images 
The local deformations of the pantograph were better captured with the two finest discretizations. The coarsest mesh was not satisfactory in that case. For the 39-th picture, which refers to the maximum applied displacement, Figure 8 (e-h) shows that the coarsest mesh did not capture at all the kinematic details of the test. The deformations were better described, even imperfectly (Figure 7), by the two finest discretizations.

\subsection{Mesoscale Results}

In Figure 9, the change of the RMS gray level residuals are reported for the three different meshes used in mesoscopic registrations (Figure 3). The average RMS residuals are all three of the order of 10 gray levels. The influence of the discretization is not as important as at the macroscale (Figure 7). Further, the two finest meshes at the macroscale led to correlation residuals that were higher (i.e., about $40 \%$ ) than those at the mesoscale. Last, let us note that all RMS "jump" observed at the macroscale also occurred in the present case for the same reasons.

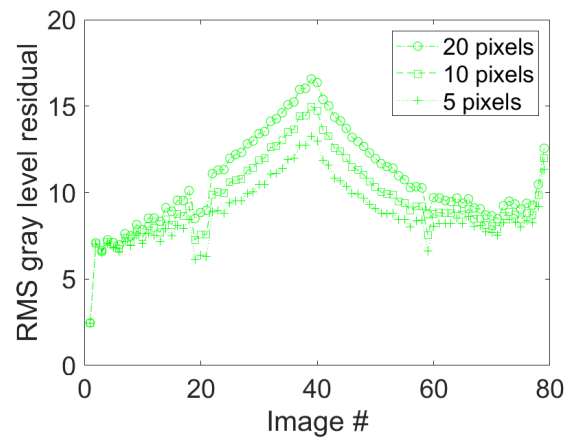

Fig. 9 RMS gray level residuals in DIC analyses at the mesoscopic scale

The quasi mesh-independence of the DIC results at the mesoscale is confirmed for the gray level residual fields computed for the 19-th picture (see Figure 10 a-c)). The differences with the macroscopic analyses (Figure $8(a-d)$ ) prove that when the mesh could be fully 
adapted to the mesostructure, the corresponding kinematics was better accounted for the experiment, especially in the vicinity of the hinges for the finest discretizations.

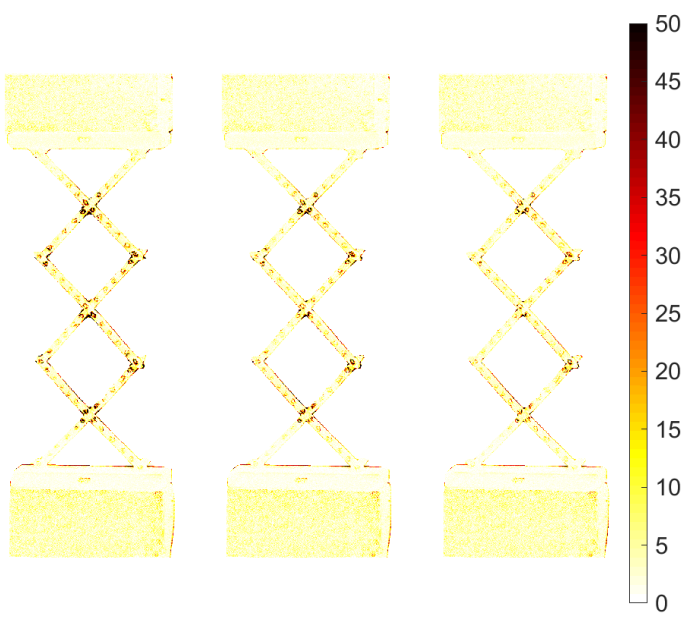
(a) $\ell=20 \mathrm{px}$
(b) $\ell=10 \mathrm{px}$
(c) $\ell=5 \mathrm{px}$

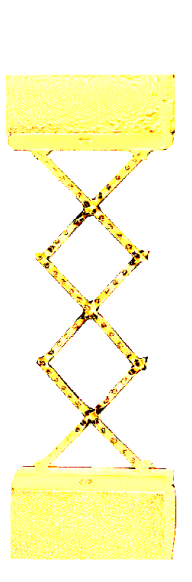

(d) $\ell=20 \mathrm{px}$

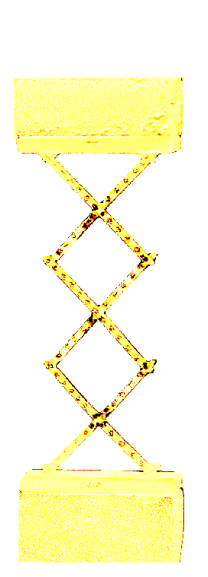

(e) $\ell=10 \mathrm{px}$

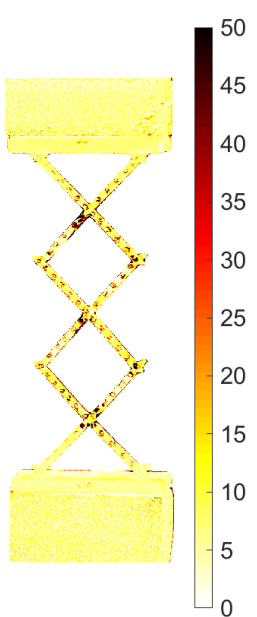

(f) $\ell=5 \mathrm{px}$

Fig. 10 Gray level residual fields for the three different meshes used in DIC analyses at the mesoscale of the 19-th (top row) and 39-th (bottom row) images

The differences between mesoscopic and macroscopic analyses are more striking for picture \#39 (see Figures 8,e-h) and 10,d-f)). The registration had failed for the macroscopic 
analysis with the coarse mesh but was better with finer discretizations. Conversely, the mesh density influenced the registrations at the mesoscale essentially in the close vicinity of the hinges. The mesoscale analyses showed that there was a clear benefit by accounting for all the geometric details of the mesostructure of such metamaterials. What was still lacking was a better kinematic description close to the hinges. The following analyses aim at addressing this point.

\subsection{Microscale Results}

For the first series of analyses at the microscale (i.e., for meshed beams, see Figure 4 (b)), the residuals were very low for the 19-th picture (Figure 11). This result shows that the selected description of the hinges was faithful. Conversely, for the 39-th picture, the residuals increased, especially close to some of the hinges. This observation proves that the hypothesis of perfect hinge is violated.

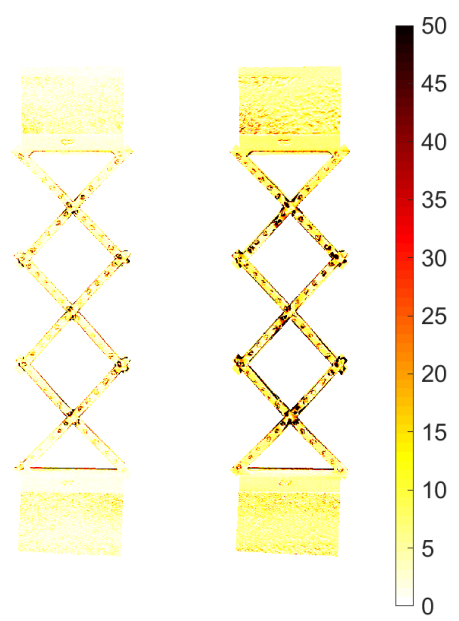

(a) (b)

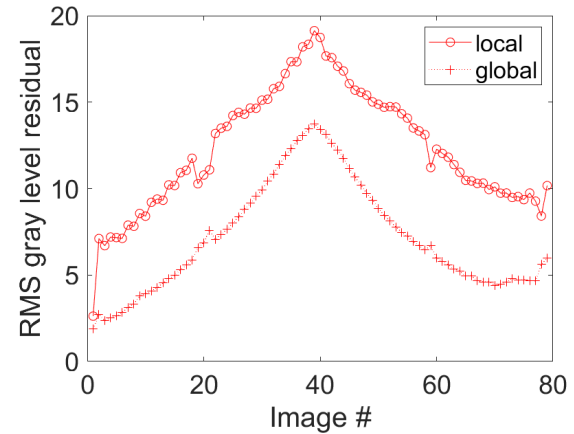

(c)

Fig. 11 Gray level residual fields for the microscopic mesh for the 19-th (a) and 39-th (b) images. (c) RMS gray level residuals for DIC analyses at the microscopic scale 
The previous trends are now summarized in Figure 11.c) that shows the change of the correlation residuals for the two analyses at the microscopic scale (Figure $4(\mathrm{~b}-\mathrm{c})$ ). For the mesh explicitly accounting for the hinges (Figure 4(b)), the average RMS residual was equal to 7.5 gray levels. This level can be compared to those observed with mesoscale analyses since the ROIs were virtually identical. These results show that the explicit modeling of the hinges leads to the best results (i.e., correlation residuals on average $25 \%$ lower in comparison with mesoscale analyses).

If discrete meso-models are to be used, the only relevant information is related to the motions of the hinges [23]. The discretization shown in Figure 4 (c) was tailored for that purpose. The corresponding residuals remained quite high (Figure 11.(c)) in comparison with all other cases. This trend is due to the fact that the actual kinematics in the close vicinity of the hinges is complex, and that the analyzed zones were precisely focussing on the hinges. This result does not mean that such analyses should be discarded. However, it calls for attention when comparing such measurements to numerical simulations.

From these first analyses, it is concluded that the macroscopic kinematics is too poor to properly capture the local deformations of the studied pantographic structure. This is especially true for coarse discretizations. The underlying model may yield limited predictions. Overall, the mesoscale kinematics better captured the actual deformations of the sample, which is proven by lower gray level residuals in comparison to the macroscopic analyses. The microscopic results are the most faithful (i.e., they lead to the lowest residuals).

\subsection{Comparison of Different Kinematic Hypotheses}

The following discussion deals with the kinematic fields. Figure 12 a-d) shows the longitudinal displacement fields, expressed in pixels (i.e., $1 \mathrm{px} \equiv 52 \mu \mathrm{m}$ ), when the applied displacement reached half the maximum level (i.e., picture \#19). 

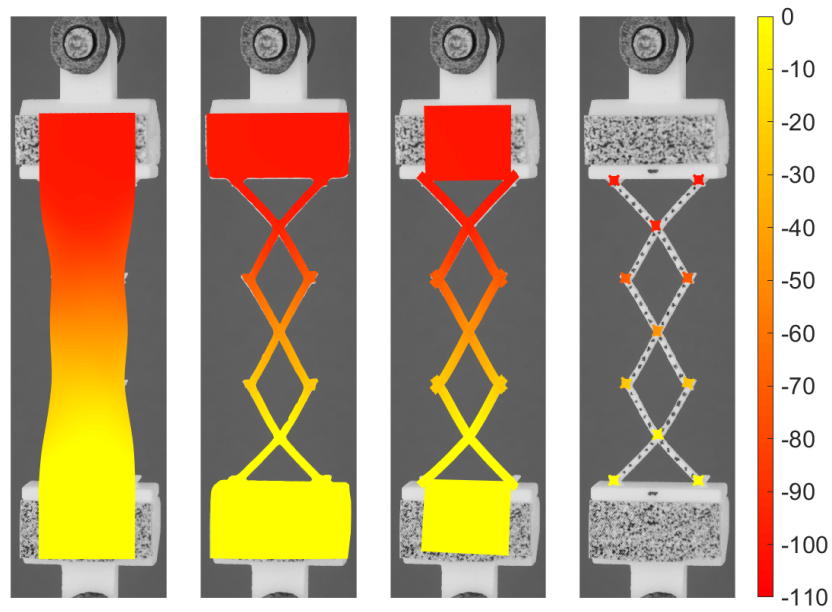

(a) macro

(b) meso

(c) micro

(d) micro

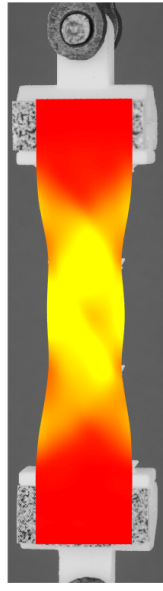

(e) macro

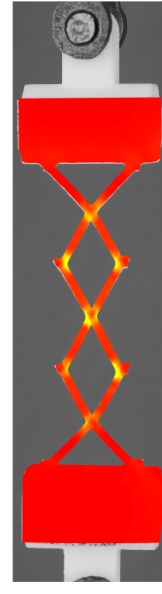

(f) meso

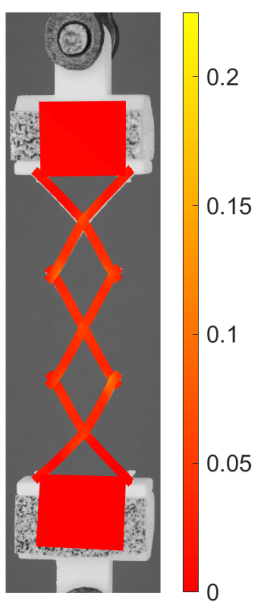

(g) micro

Fig. 12 (a-d) Longitudinal displacement fields (expressed in pixels, $1 \mathrm{px} \equiv 52 \mu \mathrm{m}$ ) for DIC analyses at various scales. (e-g) Corresponding maximum principal strain fields for the continuous approaches. The applied displacement is half its maximum level (i.e., picture \#19).

All following fields are plotted on the deformed meshes. For visual comparison purposes, the image of the deformed configuration is shown in the background. The meshes at the meso- and micro-scale follow rather well the experimental sample deformation. Sim- 
ilarly, the local analyses are following the motions of the 11 hinges. Such statements can be made qualitatively thanks to the pictures of the deformed configuration. More quantitatively, the gray level residual fields (Figures 8,10 , and 11 (c)) prove that the registrations were generally successful.

From the displacement fields, the strain fields were computed by exact differentiation of the shape functions of T3 elements. In the present case, the Green-Lagrange strain measure was selected. The maximum level of the color bars corresponds to 1.5 times the overall Green-Lagrange strain (i.e., computed from the length variation between the two supports). Figure 12 (e-g) shows the maximum principal strain field for global DIC analyses (image \#19) at different scales with approximately the same mesh size (i.e., $11 \mathrm{px}$ ). For the macroscopic mesh (Figure 12 e) ), the strains were very high over most of the ROI except in the two supports. This result was not found for the other meshes. Figure 12 f) shows that most of the strains were concentrated about the hinges, and significantly lower in the other parts. This observation applies to a lesser degree for the microscopic mesh (Figure 12 $\mathrm{g}$ )). From the residual fields, it was concluded that the strain fields at the microscopic scale were the closest to the experiment.

Figure $13(\mathrm{a}-\mathrm{d})$ shows the longitudinal displacement fields when the applied displacement reaches the maximum level. Even though the mesoscopic and microscopic registrations led to higher residuals (Figures 10 and 11 a-b)) in comparison to image \#19, the sample deformation was rather well captured. Such remark also applied for the macroscopic analyses (Figure 8). 

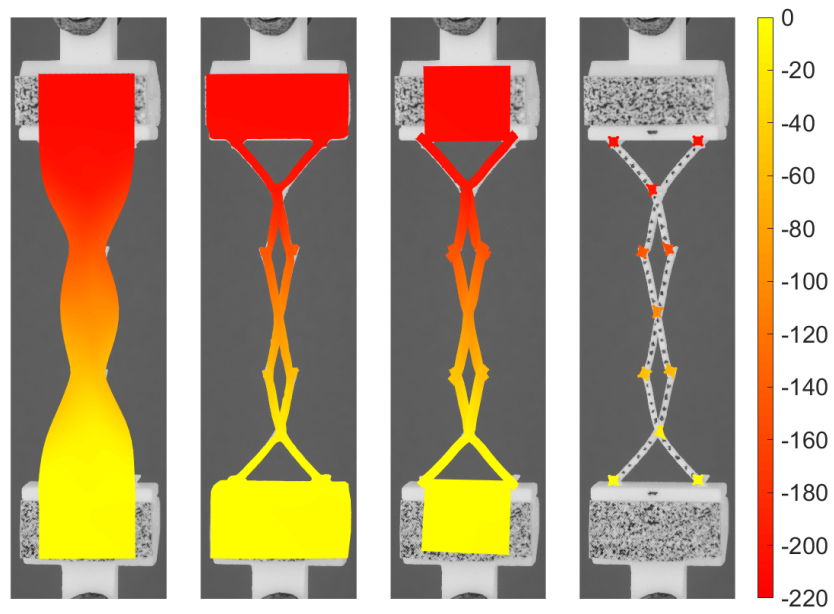

(a) macro

(b) meso

(c) micro

(d) micro

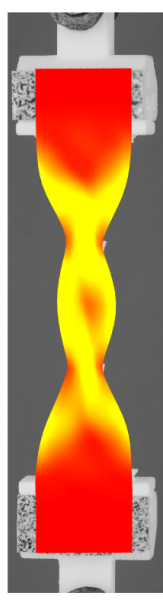

(e) macro

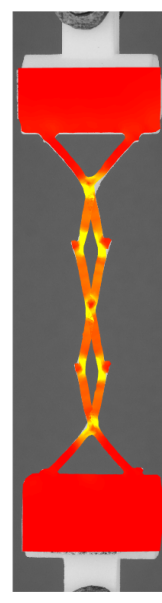

(f) meso

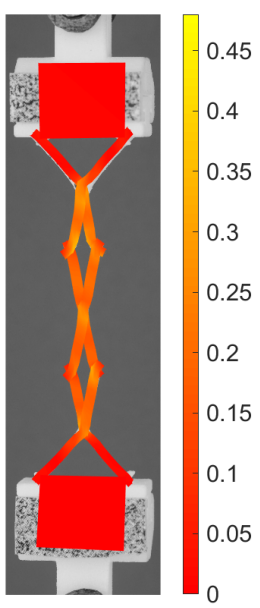

(g) micro

Fig. 13 Longitudinal displacement fields (expressed in pixels, $1 \mathrm{px} \equiv 52 \mu \mathrm{m}$ ) for DIC analyses at various scales for picture \#39 (maximum level). (e-g) Corresponding maximum principal strain fields for the continuous approaches.

For image \#39 (i.e., for the maximum applied displacement), Figure 13 (e-g) shows the maximum principal strain field for global DIC analyses at different scales. For the macroscopic mesh (Figure 13(e)), the strains were still higher than in the previous case (Fig- 
ure 12 (e)), as expected. For the other two meshes (Figure 12 f $\mathrm{f}-\mathrm{g}$ )), the strains were also concentrated in the vicinity of the hinges, but the beams also experienced significant elongations, except those that were connected to the two supports.

4.5 Analysis of the Hinges

Since the kinematic admissibility of the hinges was enforced via Lagrange multipliers $\{\boldsymbol{\lambda}\}$ for each component of the relative displacement of hinges, their level indicates whether this constraint is physically realistic or not. High levels of Lagrange multipliers thus call for attention (i.e., the pseudo-cohesive forces become very high). Figure 14 a) shows the Lagrange multiplier vector (in arbitrary unit) for the 19-th and 39-th image. Very high levels were observed for the three central hinges. The hypothesis of perfect hinge thus is questionable. For the other hinges, the amplitudes of the Lagrange vectors were lower, thereby indicating that the kinematic constraint was plausible. For the 19-th image, the overall levels were significantly smaller. In that case, the assumption of perfect hinges was better satisfied. The present analysis of experimental results therefore indicates that a two-field model [18] for pantographic sheets may be necessary.

Figure $14(b-c)$ shows the history of the horizontal and vertical components of the Lagrange multiplier vectors. The overall trend coincides with the applied displacement. The three central hinges led to the highest levels in both directions. Conversely, the Lagrange multipliers associated with the two left hinges had very small levels during the whole test. 


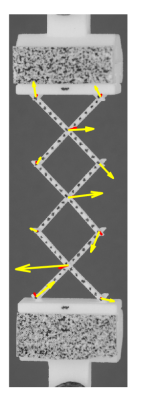

(a)

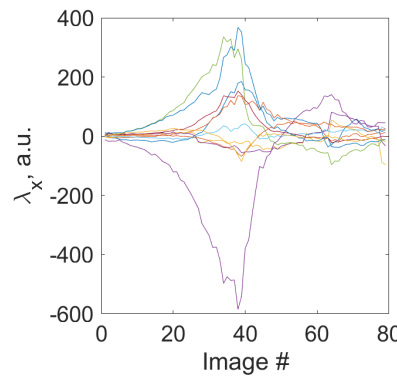

(b)

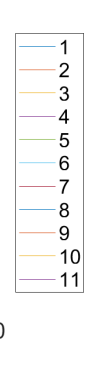

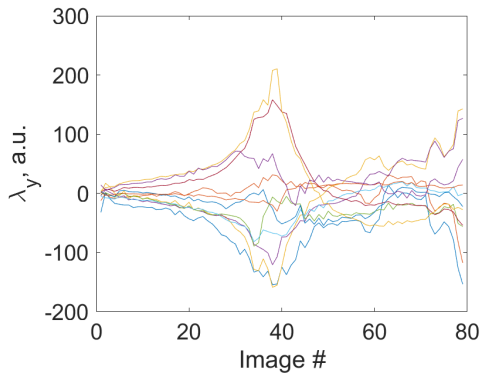

(c)

Fig. 14 (a) Lagrange multiplier vectors (in arbitrary units) for the 19-th (red arrows) and 39-th (yellow arrows) images. History of Lagrange multiplier in the vertical (b) and horizontal (c) directions. The numbers correspond to the labels of the hinges (Figure 4 a))

All these results suggest to relax the kinematic constraints on each hinge as a displacement jump may have occurred at the nodes of the two meshes that coincide for the hinge center. As a consequence, the macromodel for pantographic metamaterials would have to be formulated by using two distinct placement fields (i.e., two sets of positions corresponding to the hinges that experience relative rotations and translations), one for each beam array. Figure 15 a) shows the history of the correlation residuals with this new kinematic hypothesis (i.e., the constrains prescribed by the matrix $[\boldsymbol{L}]$ were no longer enforced in the minimization scheme $(10)$ ). When compared to the results with Lagrange multipliers (Figure 11.c)), there was clear gain when suppressing the corresponding constraints. The mean RMS residual was equal to 4.5 gray levels. This $40 \%$ decrease fully validated this last hypothesis, namely, the hinges experienced displacement jumps at their connections with the top and bottom beam layers. 


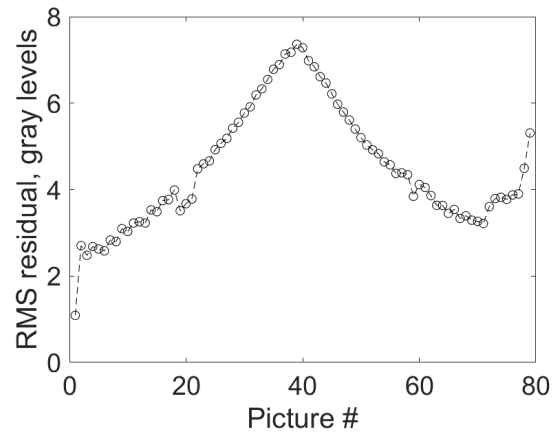

(a)

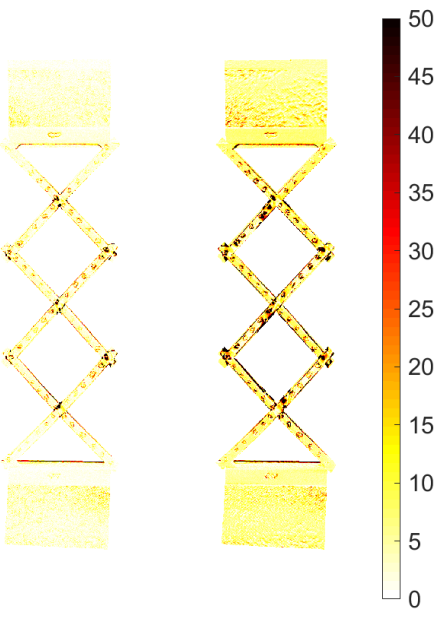

(b)

(c)

Fig. 15 (a) RMS gray level residuals for DIC analyses at the microscopic scale. Gray level residual fields for the microscopic mesh for the 19-th (b) and 39-th (c) images with no kinematic constraints at the hinges

Figure 15 (b-c) shows the gray level residuals for the 19-th and 39-th pictures. For the 19-th image, the residuals remained very low except around very small zones, some of them close to the hinges. For the 39-th image, the residuals were evenly distributed irrespective of the hinge locations. This observation further validates the new kinematic hypothesis.

Figure 16 a) shows the displacement jump vector at the 11 hinges for the 19-th and 39-th images. For the 39-th picture, as for the Lagrange multipliers, very high levels were observed for two (out of three) central hinges, which proved that the hypothesis of perfect hinge was not valid. For most of the other hinges, the amplitudes of displacement jumps were lower, yet not vanishing. For the 19-th image, the overall levels were significantly smaller. Figures 16 b-c) show the history of the horizontal and vertical components of the displacement jump vectors. The overall trend coincides with the applied displacement for the horizontal component. For the vertical component, the general trends were less obvious, which points to the fact that the experiment had no symmetry because of various imperfec- 
tions (e.g., boundary conditions, initial pantograph geometry, 2D hypotheses). All hinges experienced non-vanishing displacement jumps.

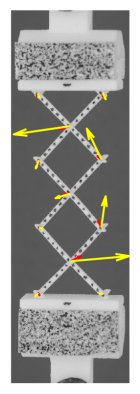

(a)

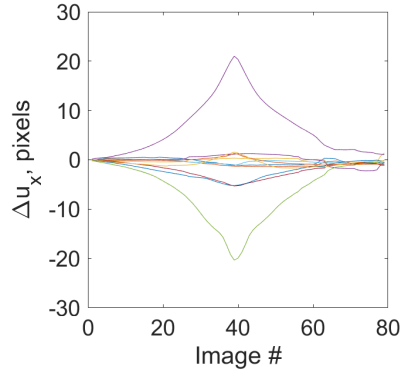

(b)
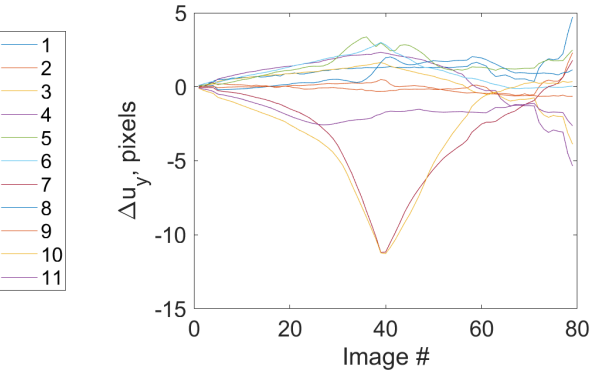

(c)

Fig. 16 (a) Displacement jump vectors for the 19-th (red arrows) and 39-th (yellow arrows) images. History of displacement jump (expressed in pixels, $1 \mathrm{px} \equiv 52 \mu \mathrm{m}$ ) in the vertical (b) and horizontal (c) directions. The numbers correspond to the labels of the hinges (Figure $4 \mathrm{a}$ ))

Figure 17 shows the maximum principal strain field for images \#19 and \#39. For the first case, the strain levels remained very low everywhere and did not concentrate around hinges. Conversely, the strains were higher in the second case, which indicates that, at that level of stretch, some strain energy was stored in the metamaterial. 


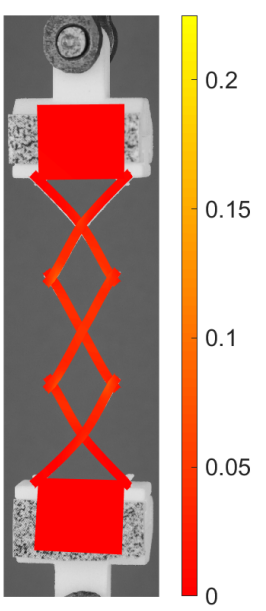

(a)

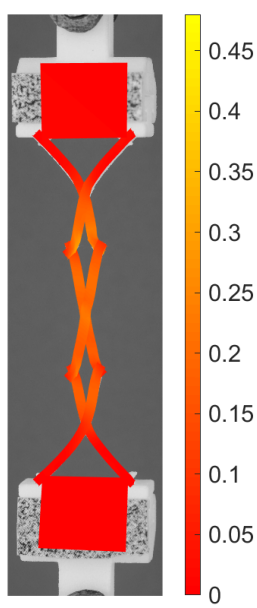

(b)

Fig. 17 Maximum principal strain for DIC analyses at the microscopic scale for the 19-th (a) and 39-th (b) images with no kinematic constraints at the hinges

\section{Conclusion}

In the present paper, the analysis of the deformations of elementary pantographic cells was studied at three different scales in a bias extension test. These cells constitute the elementary pattern of so-called pantographic metamaterials. At the macroscopic scale, the behavior of such metamaterials is expected to follow second gradient theories [7, 23, 15]. At an intermediate (meso)scale, pantographic metamaterials can be described as a set elastic extensional and rotational springs [24]. With the models introduced up to now, the details of the 2D morphology were taken into account but the deformation of the hinges was not explicitly accounted for. Conversely, at the microscale, the deformation of the deformable cylinders interconnecting the arrays of beams can be explicitly described [7]. In order to supply an experimental support to the modeling assumptions to be made for describing at all these three scales the pantographic metamaterial that was considered, different analyses were performed and their results were compared. 
It is worth noting that the microstructure of pantographic metamaterials is not classical for DIC analyses in which, usually, the material is considered as a continuum. Further, the sample was not totally patterned. Such situation required regularization strategies to be implemented. Since the material underwent very large deformations, Hencky-type regularization was selected to avoid penalizing too much the measurements. The results reported herein show the feasibility of performing DIC analyses at three different scales related to the microstructure of pantographic metamaterials. They showed that, depending on the measurement scale, the kinematics was more or less trustworthy in comparison with the studied experiment. Such type of analyses can be extended and compared to numerical simulations of various models. The gray level residuals will then constitute an objective way of probing each model with respect to the image set acquired during experiments.

The morphology of the pantographic cells and their kinematics made DIC analyses very challenging as evidenced by gray level residuals that were very high in comparison to regular cases. Such observation does not mean that DIC was not applicable. However, it probes whether various kinematic hypotheses were consistent with the experimental data (i.e., the set of images acquired during the bias extension test). The first analysis at the microscale was obtained by prescribing kinematic compatibility between the top and bottom beams via Lagrange multipliers. The second one relaxed such constraints and enable displacement jumps to be evaluated for each hinge. This last path led to the best results (i.e., the lower correlation residuals). A local analysis tracking only the mean displacement of hinges was also carried out. This analysis will guide the derivation of discrete models. Other kinematic hypotheses may be considered in future DIC studies such as those provided by beam elements [10].

The experimental quantifications reported herein give important indications about the mechanical behavior of the considered pantographic cells and supply important indications for future modeling efforts. It was proven that around the interconnecting circular cylinders 
(modeled as hinges at the microscale), relative displacements occur. They are related to non-negligible deformation energy. Consequently, the two-beam layers in a pantographic metamaterial must, in some specific situations, have to be described at the macrolevel by a two-field continuum model. This conclusion supports the assumptions made by Spagnuolo et al. [18].

Acknowledgements AM is supported by the United States National Science Foundation grant CMMI1727433. FdI acknowledges the financial support by MEMOCS Centre Università dell'Aquila (Laboratorio Prove Materiali).

\section{Compliance with Ethical Standards}

The authors have no conflict of interest to declare.

\section{References}

1. T. Archer, P. Beauchene, C. Huchette, and F. Hild. Global digital image correlation up to very high temperatures with grey level corrections. Measurement Science and Technology, 31(2):024003, 2020.

2. A. Charbal, J.-E. Dufour, A. Guery, F. Hild, S. Roux, L. Vincent, and M. Poncelet. Integrated digital image correlation considering gray level and blur variations: Application to distortion measurements of ir camera. Optics and Lasers in Engineering, 78:75-85, 2016.

3. D. Claire, F. Hild, and S. Roux. A finite element formulation to identify damage fields: The equilibrium gap method. Int. J. Num. Meth. Engng., 61(2):189-208, 2004.

4. F. dell'Isola, P. Seppecher, J.-J. Alibert, T. Lekszycki, R. Grygoruk, M. Pawlikowski, D. Steigmann, I. Giorgio, U. Andreaus, E. Turco, M. Golaszewski, N. Rizzi, C. Boutin, V.A. Eremeyev, A. Misra, L. Placidi, E. Barchiesi, L. Greco, M. Cuomo, A. Cazzani, A.D. Corte, A. Battista, D. Scerrato, I. Zurba Eremeeva, Y. Rahali, J.-F. Ganghoffer, W. Müller, G. Ganzosch, M. Spagnuolo, A. Pfaff, K. Barcz, K. Hoschke, J. Neggers, and F. Hild. Pantographic metamaterials: an example of mathematically driven design and of its technological challenges. Continuum Mechanics and Thermodynamics, 31(4):851-884, 2019.

5. F. dell'Isola, P. Seppecher, M. Spagnuolo, E. Barchiesi, F. Hild, T. Lekszycki, I. Giorgio, L. Placidi, U. Andreaus, M. Cuomo, S.R. Eugster, A. Pfaff, K. Hoschke, R. Langkemper, E. Turco, R. Sarikaya, 
A. Misra, M. De Angelo, F. D’Annibale, A. Bouterf, X. Pinelli, A. Misra, B. Desmorat, M. Pawlikowski, C. Dupuy, D. Scerrato, P. Peyre, M. Laudato, L. Manzari, P. Göransson, C. Hesch, S. Hesch, P. Franciosi, J. Dirrenberger, F. Maurin, Z. Vangelatos, C. Grigoropoulos, V. Melissinaki, M. Farsari, W. Muller, E. Abali, C. Liebold, G. Ganzosch, P. Harrison, R. Drobnicki, L.A. Igumnov, F. Alzahrani, and T. Hayat. Advances in Pantographic Structures: Design, Manufacturing, Models, Experiments and Image Analyses. Continuum Mechanics and Thermodynamics, 31(4):1231-1282, 2019.

6. C. Geuzaine and J.-F. Remacle. Gmsh: a three-dimensional finite element mesh generator with builtin pre- and post-processing facilities. International Journal for Numerical Methods in Engineering, 79(11):1309-1331, 2009.

7. I. Giorgio. Numerical identification procedure between a micro-cauchy model and a macro-second gradient model for planar pantographic structures. Zeitschrift für angewandte Mathematik und Physik, 67(4):95, 2016.

8. I. Giorgio, N.L. Rizzi, and E. Turco. Continuum modelling of pantographic sheets for out-of-plane bifurcation and vibrational analysis. Proceedings of the Royal Society A, 473:20170636, 2017.

9. F. Hild and S. Roux. Digital image correlation. In P. Rastogi and E. Hack, editors, Optical Methods for Solid Mechanics. A Full-Field Approach, pages 183-228, Weinheim (Germany), 2012. Wiley-VCH.

10. F. Hild, S. Roux, R. Gras, N. Guerrero, M.E. Marante, and J. Flórez-López. Displacement measurement technique for beam kinematics. Optics and Lasers in Engineering, 47:495-503, 2009.

11. H. Leclerc, J. Neggers, F. Mathieu, F. Hild, and S. Roux. Correli 3.0. IDDN.FR.001.520008.000.S.P.2015.000.31500, Agence pour la Protection des Programmes, Paris (France), 2015.

12. H. Leclerc, J.N. Périé, S. Roux, and F. Hild. Integrated digital image correlation for the identification of mechanical properties. In A. Gagalowicz and W. Philips, editors, MIRAGE 2009, volume LNCS 5496, pages 161-171, Berlin (Germany), 2009. Springer.

13. A. Misra, T. Lekszycki, I. Giorgio, G. Ganzosch, W.H. Müller, and F. dell'Isola. Pantographic metamaterials show atypical poynting effect reversal. Mechanics Research Communications, 89:6-10, 2018.

14. N. Nejadsadeghi, M. De Angelo, R. Drobnicki, T. Lekszycki, F. dell'Isola, and A. Misra. Parametric Experimentation on Pantographic Unit Cells Reveals Local Extremum Configuration. Experimental Mechanics, 59:927-939, 2019.

15. L. Placidi, U. Andreaus, and I. Giorgio. Identification of two-dimensional pantographic structure via a linear D4 orthotropic second gradient elastic model. Journal of Engineering Mathematics, 103:1-21, 
2017.

16. J. Réthoré, S. Roux, and F. Hild. An extended and integrated digital image correlation technique applied to the analysis fractured samples. European Journal of Computational Mechanics, 18:285-306, 2009.

17. S. Roux, F. Hild, and H. Leclerc. Mechanical assistance to dic. In H. Espinosa and F. Hild, editors, Full field measurements and identification in Solid Mechanics, volume Procedia IUTAM, 4, pages 159-168. Elsevier, 2012.

18. M. Spagnuolo, K. Barcz, A. Pfaff, F. dell'Isola, and P. Franciosi. Qualitative pivot damage analysis in aluminum printed pantographic sheets: Numerics and experiments. Mechanics Research Communications, 83:47-52, 2017.

19. M.A. Sutton. Computer vision-based, noncontacting deformation measurements in mechanics: A generational transformation. Applied Mechanics Reviews, 65(AMR-13-1009):050802, 2013.

20. M.A. Sutton, J.J. Orteu, and H. Schreier. Image correlation for shape, motion and deformation measurements: Basic Concepts, Theory and Applications. Springer, New York, NY (USA), 2009.

21. A.N. Tikhonov and V.Y. Arsenin. Solutions of ill-posed problems. J. Wiley, New York (USA), 1977.

22. Z. Tomičević, F. Hild, and S. Roux. Mechanics-aided digital image correlation. Journal of Strain Analysis for Engineering Design, 48:330-343, 2013.

23. E. Turco, F. dell'Isola, A. Cazzani, and N.L. Rizzi. Hencky-type discrete model for pantographic structures: numerical comparison with second gradient continuum models. Zeitschrift für angewandte Mathematik und Physik, 67:85, 2016.

24. E. Turco, M. Golaszewski, A. Cazzani, and N.L. Rizzi. Large deformations induced in planar pantographic sheets by loads applied on fibers: Experimental validation of a discrete Lagrangian model. Mechanics Research Communications, 76:51-56, 2016.

25. E. Turco, M. Golaszewski, I. Giorgio, and F. D’Annibale. Pantographic lattices with non-orthogonal fibres: Experiments and their numerical simulations. Composites Part B, 118:1-14, 2017.

26. E. Turco, A. Misra, M. Pawlikowski, F. dell'Isola, and F. Hild. Enhanced piola-hencky discrete models for pantographic sheets with pivots without deformation energy: Numerics and experiments. International Journal of Solids and Structures, 147:94-109, 2018.

27. E. Turco, A. Misra, R. Sarikaya, and T. Lekszycki. Quantitative analysis of deformation mechanisms in pantographic substructures: experiments and modeling. Continuum Mechanics and Thermodynamics, 31:209-233, 2019. 